九州大学学術情報リポジトリ

Kyushu University Institutional Repository

\title{
MODELING OF NERVOUS SYSTEMS BY DOUBLY STOCHASTIC POISSON PROCESSES
}

\section{Kano, Seigo}

Research Institute of Fundamental Information Science, Kyushu University

Shigenaga, Shin-ichi

Department of Information Systems, Graduate School of Engineering Science, Kyushu University

https://doi.org/10.5109/13335

出版情報: Bulletin of informatics and cybernetics. 20 (1/2)，pp.43-54，1982-03. Research Association of Statistical Sciences

バージョン :

権利関係 : 


\title{
MODELING OF NERVOUS SYSTEMS BY DOUBLY STOCHASTIC POISSON PROCESSES
}

\author{
By
}

\author{
Seigo KANo $\overline{0}^{*}$ and Shin-ichi ShIGENAGA**
}

(Received November 18, 1981)

\begin{abstract}
Neuron impulse sequences are represented by doubly stochastic Poisson Processes and Kalman filtering theory is applied to estimate the random intensity functions of the processes. Models of nervous systems in which exitatory and inhibitory synapses have elementary operation, are studied by simultaneous states equations in the theory. The method of estimation is applied to neuron impulse sequences simultaneously recorded from preoptic area of a monkey by a microelectrode.
\end{abstract}

\section{Introduction}

In recent years, physiological experiments with micro-electrode method have made it clear that information transmission and processing in the brain are carried out through neuron impulse sequences. However we can not identify the operation of a neuron impulse sequence only by the shapes or patterns of the sequence apart from original neuron. Each neuron impulse sequence fired from an original neuron has two roles. One is function of the neuron in brain and the other is concerned with the information about activity of the neuron. For example, positions of auditory and visual areas are different in brain and neurons in their areas have proper roles about auditory or visual sense, but shapes of their neuron impulse sequences may be equal. This fact makes it possible to integrate neural information through corresponding neuron impulse sequences. Observed impulse sequences in experiments show somewhat complicated patterns which are based on their impulse frequencies. To study nervous systems we have to consider the frequencies of impulse sequences as basic variables of the system.

In this paper, some mathematical models of nervous systems are given and estimation for time dependent fluctuation of impulse frequencies is performed on the models.

For analysis of nervous systems, N. Wiener's stochastic nonlinear theory [1] has been used by the paper [2] and the others. The theory gives powerful tool for continuous data of nerve action potential or brain waves. For discrete data of neuron impulse sequences we had no method to analyze. We apply Kalman filtering theory

* Research Institute of Fundamental Information Science, Kyushu University 33, Fukuoka 812, Japan.

** Department of Information Systems, Graduate School of Engineering Science, Kyushu University 39, Fukuoka 812, Japan. 
to random intensity functions of doubly stochastic Poisson processes which are models of neuron impulse sequences. The method of this paper is considered to be valuable for analyses of nervous systems by the impulse sequence and to increase the effectiveness of physiological experiments with micro-electrodes still more.

\section{Stochastic models of neuron impulse sequences}

We assume that neuron impulse sequence conducted by each axon in a nervous system forms a stochastic point process termed neuron point process in this paper. We have neuron input and output point processes for input and output impulse sequences of a nervous system denoted by $\left\{M_{t} ; t \geqq t_{0}\right\}$ and $\left\{N_{t} ; t \geqq t_{0}\right\}$, respectively; or $\left\{M_{t}\right\}$ and $\left\{N_{t}\right\}$, for abbreviation. For multi-dimensional input and output point processes, we use $\boldsymbol{M}_{t}=\left(M_{t}^{(1)}, \cdots, M_{t}^{(m)}\right)^{\prime 1)}$ and $\boldsymbol{N}_{t}=\left(N_{t}^{(1)}, \cdots, N_{t}^{(n)}\right)^{\prime}$, respectively. Let intensity of neuron point process correspond to frequency of neuron impulse sequence and we denote those of input and output one-dimensional point processes by $\pi_{t}$ and $\lambda_{t}$, and $\Pi_{t}=\left(\pi_{t}^{(1)}, \cdots, \pi_{t}^{(m)}\right)^{\prime}$ and $\Lambda_{t}=\left(\lambda_{t}^{(1)}, \cdots, \lambda_{t}^{(n)}\right)^{\prime}$, for multi-dimensional point processes, respectively. In this paper, neuron point processes are assumed to be doubly stochastic Poisson processes, which are defined as follows according to [3].

DOUBLY STOCHASTIC POISSON PROCESS. A stochastic point process $\left\{\Lambda_{t} ; t \geqq t_{0}\right\}$ is a doubly stochastic Poisson process with intensity process $\left\{\lambda_{t}\left(\boldsymbol{x}_{t}\right) ; t \geqq t_{0}\right\}$ if conditionally stochastic point process $\left\{N_{t}\right\}$ for given stochastic process $\left\{\boldsymbol{x}_{t} ; t \geqq t_{0}\right\}$ is a Poisson process with intensity $\lambda_{t}\left(\boldsymbol{x}_{t}\right) . \quad N$-dimensional doubly stochastic Poisson process $\left\{\boldsymbol{N}_{t} ; t \geqq t_{0}\right\}$ with intensity process $\left\{\Lambda_{t}\left(\boldsymbol{x}_{t}\right) ; t \geqq t_{0}\right\}$ is a stochastic point process such that its component processes $\left\{N_{t}^{(i)} ; t \geqq t_{0}\right\}, i=1, \cdots, n$, are doubly stochastic Poisson processes with intensity processes $\left\{\lambda_{t}^{(i)}\left(\boldsymbol{x}_{\imath}\right) ; t \geqq t_{0}\right\}, i=1, \cdots, n$, respectively, and are conditionally independent for given stochastic process $\left\{\boldsymbol{x}_{t} ; t \geqq t_{0}\right\}$.

From this definition we have

$$
\mathrm{E}\left(N_{t}\right)=\mathrm{E}\left\{\lambda_{t}\left(\boldsymbol{x}_{t}\right)\right\}, \quad \mathrm{E}\left(\boldsymbol{N}_{t}\right)=\mathrm{E}\left\{\Lambda_{t}\left(\boldsymbol{x}_{t}\right)\right\} .
$$

\section{Modeling of nervous systems}

We assume that stochastic process $\left\{\boldsymbol{x}_{t}\right\}$ which gives the intensity function $\Lambda_{t}\left(\boldsymbol{x}_{t}\right)$ of doubly stochastic Poisson process is intensity function of input neuron point process. Then, output of a nervous system is doubly stochastic Poisson process with intensity $\Lambda_{t}\left(\boldsymbol{x}_{t}\right)$ and structure of the nervous system is represented by a relation of $\left\{\boldsymbol{x}_{t}\right\}$ or an equation of $\left\{\boldsymbol{x}_{t}\right\}$. It is reasonable that as the relation stochastic differential equations are applied. Using the definition of Kalman filtering theory, the differential equation is called states equation and $\left\{\boldsymbol{x}_{t}\right\}$ called states. Fitness of the equation to the nervous system is studied by estimation in which inputs $\left\{\boldsymbol{x}_{t}\right\}$ are estimated by output data $\left\{\boldsymbol{N}_{t}\right\}$.

\subsection{Estimation of $\Pi_{t}$.}

It is assumed that intensity $\Pi_{t}$ of input.process $\left\{\boldsymbol{M}_{t}\right\}$ is stochastic process which satisfies the stochastic differential equation,

1) $M^{\prime}$ represents transpose of vector or matrix $M$. 


$$
d \Pi_{t}=A_{t} \Pi_{t} d t+\boldsymbol{b}_{t} d t+\boldsymbol{u}_{t} d t, \quad \Pi_{t_{0}}=\Pi_{0},
$$

and intensity $A_{t}$ of output process $\left\{\boldsymbol{N}_{t}\right\}$ is given by the stochastic equation,

$$
\Lambda_{t}=S_{t} \Pi_{t}+\boldsymbol{h}_{0}
$$

where

$A_{t} ;$ known $m \times m$ matrix,

$\boldsymbol{b}_{t} ;$ known $m$-dimensional vector,

$\Pi_{0} ; m$-dimensional random variable with known mean $\bar{\Pi}_{0}$ and known covariance matrix $\Sigma_{0}$

$\boldsymbol{u}_{t} ; m$-dimensional normal white noise with zero-mean and covariance matrix $\mathrm{E}\left(\boldsymbol{u}_{t} \boldsymbol{u}_{\tau}^{\prime}\right)=U_{t} \delta(t-\tau)$

$S_{t} ;$ known $n \times m$ matrix,

$\boldsymbol{h}_{0}$; known $n$-dimensional constant vector.

Then, following assertion is shown.

ASSERTION 1 (estimation of $\Pi_{t}$.) Let $\Pi_{t}^{t}$ be a linear estimate of $\Pi_{t}$ in terms of output data $\left\{\boldsymbol{N}_{t}\right\}$. Denote the linear estimate $\Pi_{t}^{l}$ that minimizes error covariance matrix $\mathrm{E}\left[\left(\Pi_{t}-\Pi_{t}^{t}\right)\left(\Pi_{t}-\Pi_{t}^{t}\right)^{\prime}\right]$ by $\Pi_{t}^{*}$ : here the minimization means that $\mathrm{E}\left[\left(\Pi_{t}-\Pi_{t}^{t}\right)\left(\Pi_{t}-\Pi_{t}^{l}\right)^{\prime}\right]-$ $\mathrm{E}\left[\left(\Pi_{t}-\Pi_{t}^{*}\right)\left(\Pi_{t}-\Pi_{t}^{*}\right)^{\prime}\right]$ is nonnegative definite for all choices $\Pi_{t}^{l}$. Then, using Kalman filtering theory in [4], we can obtain the estimate $\Pi_{t}^{*}$ by following equations:

$$
\begin{aligned}
& d \tilde{\Pi}_{t}^{*}=\tilde{A}_{t} \tilde{\Pi}_{t}^{*} d t+\widetilde{\Sigma}_{t} \widetilde{C}_{t}^{\prime} \operatorname{diag}\left(\mathrm{E}\left(\lambda_{t}^{(i)}\right)\right)^{-1}\left[d \boldsymbol{N}_{t}-\tilde{C}_{t} \tilde{\Pi}_{t}^{*} d t\right], \quad \tilde{\Pi}_{t_{0}}^{*}=\left(\bar{\Pi}_{0}^{\prime}, 1\right)^{\prime}, \\
& d \widetilde{\Sigma}_{t}=\left(\tilde{U}_{t}+\tilde{A}_{t} \widetilde{\Sigma}_{t}+\widetilde{\Sigma}_{t} \tilde{A}_{t}^{\prime}-\widetilde{\Sigma}_{t} \tilde{C}_{t}^{\prime} \operatorname{diag}\left(\mathrm{E}\left(\lambda_{t}^{(i)}\right)\right)^{-1} \tilde{C}_{t} \widetilde{\Sigma}_{t}\right) d t, \quad \widetilde{\Sigma}_{t_{0}}=\widetilde{\Sigma}_{0},
\end{aligned}
$$

where

$$
\begin{aligned}
& \tilde{A}_{t}=\left(\begin{array}{ll}
A_{t} & \boldsymbol{b}_{t} \\
0 & 0
\end{array}\right) ;(m+1) \times(m+1) \text { matrix, } \\
& \tilde{\Pi}_{t}^{*}=\left(\Pi_{t}^{*^{\prime}}, 1\right)^{\prime} ;(m+1) \text {-dimensional vector, } \\
& \tilde{C}_{t}=\left(S_{t}, \boldsymbol{h}_{0}\right) ; n \times(m+1) \text { matrix, } \\
& \tilde{U}_{t}=\left(\begin{array}{ll}
U_{t} & 0 \\
0 & 0
\end{array}\right) ;(m+1) \times(m+1) \text { matrix, } \\
& \tilde{\Sigma}_{0}=\left(\begin{array}{ll}
\Sigma_{0} & 0 \\
0 & 0
\end{array}\right) ;(m+1) \times(m+1) \text { matrix, } \\
& \widetilde{\Sigma}_{t}=\left(\begin{array}{ll}
\Sigma_{t} & 0 \\
0 & 0
\end{array}\right) ;(m+1) \times(m+1) \text { matrix, } \\
& \Sigma_{t} ; \text { error covariance matrix of } \Pi_{t}^{*} .
\end{aligned}
$$

The assertion is shown as follows.

Equation (3.1) is rewritten as

$$
d \tilde{\Pi}_{t}=\tilde{A}_{t} \tilde{\Pi}_{t} d t+\tilde{\boldsymbol{u}}_{t} d t, \quad \tilde{\Pi}_{t_{0}}=\left(\Pi_{0}^{\prime}, 1\right)^{\prime},
$$


where

$$
\tilde{\Pi}_{t}=\left(\Pi_{t}^{\prime}, 1\right)^{\prime}, \quad \tilde{\boldsymbol{u}}_{t}=\left(\boldsymbol{u}_{t}^{\prime}, 0\right)^{\prime}
$$

Here, (3.4) is states equation of Kalman filter. Next, we give an observed process of the states by

$$
\boldsymbol{r}_{t}=\tilde{C}_{t} \tilde{\Pi}_{t}+\boldsymbol{w}_{t}
$$

where $\left\{\boldsymbol{w}_{t}\right\}$ is $n$-dimensional normal white noise process and independent of $\left\{\boldsymbol{u}_{t}\right\}$ such that $\mathrm{E}\left(\boldsymbol{w}_{t}\right)=\mathbf{0}$ and $\mathrm{E}\left(\boldsymbol{w}_{t} \boldsymbol{w}_{\tau}^{\prime}\right)=\operatorname{diag}\left(\mathrm{E}\left(\lambda_{t}^{(i)}\right)\right) \delta(t-\tau)$. From (3.2) and (3.5),

$$
\mathrm{E}\left(\boldsymbol{r}_{t} d t\right)=\mathrm{E}\left(\Lambda_{t}+\boldsymbol{w}_{t}\right) d t .
$$

Since $E\left(\boldsymbol{w}_{t}\right)=\mathbf{0}$, we have from (2.1) that

$$
\mathrm{E}\left(\boldsymbol{r}_{t} d t\right)=\mathrm{E}\left(d \boldsymbol{N}_{t}\right) .
$$

And from (3.2), (3.5) and independency of $\boldsymbol{w}_{t}$ and $A_{t}$,

$$
\mathrm{E}\left[\left(\boldsymbol{r}_{t} d t\right)\left(\boldsymbol{r}_{t} d t\right)^{\prime}\right]=\mathrm{E}\left[\left(\Lambda_{t} d t\right)\left(\Lambda_{t} d t\right)^{\prime}\right]+\mathrm{E}\left[\left(\boldsymbol{w}_{t} d t\right)\left(\boldsymbol{w}_{t} d t\right)^{\prime}\right] .
$$

Here, from the fact that normal white noise $\boldsymbol{w}_{t}$ can be expressed as

$$
\boldsymbol{w}_{t}=\mathrm{E}\left(\boldsymbol{w}_{t}\right)+\operatorname{diag}\left(\mathrm{E}\left(\lambda_{t}^{(i)}\right)\right)^{1 / 2} \frac{d}{d t} \boldsymbol{\beta}_{t},
$$

where $\left\{\boldsymbol{\beta}_{t}\right\}$ is $n$-dimensional Brownian motion process, we have

$$
\mathrm{E}\left[\left(\boldsymbol{w}_{t} d t\right)\left(\boldsymbol{w}_{t} d t\right)\right]^{\prime}=\operatorname{diag}\left(\mathrm{E}\left(\lambda_{i}^{(i)}\right)\right) d t .
$$

Furthermore, since from definition in Section $2\left\{\boldsymbol{N}_{t}\right\}$ is conditionally Poisson process and its component processes $\left\{N_{t}^{(i)}\right\}, i=1, \cdots, n$, are conditionally independent, we can get

$$
\mathrm{E}\left[\left(\lambda_{t} d t\right)\left(A_{t} d t\right)\right]^{\prime}+\operatorname{diag}\left(\mathrm{E}\left(\lambda_{t}^{(i)}\right)\right) d t=\mathrm{E}\left[\left(d \boldsymbol{N}_{t}\right)\left(d \boldsymbol{N}_{t}\right)^{\prime}\right] .
$$

Therefore, we have from (3.6), (3.7) and (3.8) that

$$
\operatorname{Cov}\left(\boldsymbol{r}_{t} d t\right)=\operatorname{Cov}\left(d \boldsymbol{N}_{t}\right) \text {. }
$$

Consequently, from (3.6) and (3.9), mean and covariance matrix of $\boldsymbol{r}_{t} d t$ coincide with those of $d \boldsymbol{N}_{t}$. Thus we can use observed value $d \boldsymbol{N}_{t}$ as $\boldsymbol{r}_{t} d t$ in applying Kalman filtering theory to (3.4) and (3.5). That is, the estimate $\Pi_{t}^{*}$ of $\Pi_{t}$ in terms of output data $\left\{\boldsymbol{N}_{t}\right\}$ is given by equations (3.3).

\subsection{Estimation of parameters in model systems}

It is assumed that intensity $\Pi_{t}$ of input point process is given by a stochastic differential equation,

$$
d \Pi_{t}=A_{t} \Pi_{t} d t+\boldsymbol{b}_{t} d t+\boldsymbol{u}_{t} d t, \quad \Pi_{t_{0}}=\Pi_{0},
$$

where 
$A_{t}=\left(a_{t}^{(i j)}\right), i, j=1, \cdots, m$; unknown $m \times m$ matrix

$\boldsymbol{b}_{\boldsymbol{t}}=\left(b_{t}^{(1)}, \cdots, b_{t}^{(m)}\right)^{\prime}$; unknown $m$-dimensional vector,

$\boldsymbol{u}_{t}=\left(u_{t}^{(1)}, \cdots, u_{t}^{(m)}\right)^{\prime} ; u_{t}^{(i)}, i=1, \cdots, m$, are mutually independent normal white noise with zero-mean and covariance $E\left\{u_{t}^{(i)} u_{\tau}^{(i)^{\prime}}\right\}=V_{u}^{(i)}(t) \delta(t-\tau)$, respectively,

$\Pi_{0} ; m$-dimensional random variable with known mean $\bar{\Pi}_{0}$ and known covariance matrix $\Sigma_{0}$.

Then, following assertion is shown.

AssERTION 2 (estimation of parameters.) Assume that parameters $a_{t}^{(i j)}$ and $b_{i}^{(i)}$ are constant value, and that observed value $\tilde{\pi}_{t}^{(i)}$ of $i$-component $\pi_{t}^{(i)}$ of $\Pi_{t}$ is given by

$$
\tilde{\pi}_{t}^{(i)}=\pi_{t}^{(i)}+w_{t}^{(i)}, \quad i=1, \cdots, m,
$$

where $w_{t}^{(i)}$ is normal white noise with zero-mean and covariance $E\left\{w_{t}^{(i)} w_{t}^{(i)^{\prime}}\right\}=V_{w}^{(i)}(t) \hat{\partial}(t-\tau)$, respectively. Then mean-square estimates $a_{t}^{*(i j)}$ and $b_{t}^{*(i)}$ of parameters $a_{t}^{(i j)}$ and $b_{t}^{(i)}$ in terms of observed value of $\left\{\Pi_{t}\right\}$ are given by following equations:

$$
\begin{aligned}
d \tilde{K}_{t}^{*(i)} & =\Sigma_{t}^{(i)} C_{t}^{\prime} V_{v}^{(i)}(t)^{-1}\left[d \tilde{\pi}_{t}^{(i)}-C_{t} \tilde{K}_{t}^{*(i)} d t\right], \quad i=1, \cdots, m, \\
\Sigma_{t}^{(i)} & =-\left(\Sigma_{t}^{(i)} C_{t}^{\prime} V_{v=}^{(i)}(t)^{-1} C_{t} \Sigma_{t}^{(i)}\right) d t, \quad i=1, \cdots, m,
\end{aligned}
$$

where

$$
\begin{aligned}
\tilde{K}_{t}^{*(i)} & =\left(a_{t}^{*(i 1)}, \cdots, a_{t}^{*(i m)}, b_{t}^{*(i)}\right)^{\prime}, \\
C_{t} & =\left\langle\tilde{\pi}_{t}^{(1)}, \cdots, \tilde{\pi}_{t}^{(m)}, 1\right), \\
V_{v}^{(i)}(t) & =V_{u}^{(i)}(t)+\operatorname{Var}\left(d w_{t}^{(i)} / d t\right)+\sum_{j=1}^{m}\left[\left(a_{t}^{(i j)}\right)^{2} V_{w}^{(j)}(t)\right] .
\end{aligned}
$$

The assertion is shown as follows.

Rearranging (3.10) with regard to $i$-component of $\Pi_{t}$, we have following $m$ equations :

$$
d \pi_{t}^{(i)}=\left(\pi_{t}^{(1)}, \cdots, \pi_{t}^{(m)}, 1\right)\left(a_{t}^{(i 1)}, \cdots, a_{t}^{(i m)}, b_{t}^{(i)}\right)^{\prime} d t+u_{t}^{(i)} d t, \quad i=1, \cdots, m .
$$

Using equations (3.11), we have

$$
d \tilde{\pi}_{t}^{(i)}=\left(\tilde{\pi}_{t}^{(1)}, \cdots, \tilde{\pi}_{t}^{(m)}, 1\right)\left(a_{t}^{(i)}, \cdots, a_{t}^{(i m)}, b_{t}^{(i)}\right)^{\prime} d t+v_{t}^{(i)} d t, \quad i=1, \cdots, m,
$$

where

$$
v_{t}^{(i)}=u_{t}^{(i)}+d w_{t}^{(i)} / d t-\sum_{j=1}^{m} a_{t}^{(i j)} w_{t}^{(j)}, \quad i=1, \cdots, m .
$$

Since parameters $a_{t}^{(i j)}$ and $b_{t}^{(i)}$ are constant,

$$
\frac{d}{d t}\left(\begin{array}{c}
\boldsymbol{a}_{t}^{(i)} \\
b_{t}^{(i)}
\end{array}\right)=\mathbf{0}, \quad i=1, \cdots, m,
$$

where $\boldsymbol{a}^{(i)}=\left(a_{t}^{(i 1)}, \cdots, a_{t}^{(i m)}\right)^{\prime}$. Applying Kalman filtering theory to (3.14) and (3.15), we have equations (3.12). 


\subsection{Estimation of $\Lambda_{t}$}

It is assumed that output process $\left\{\boldsymbol{N}_{t}\right\}$ is $n$-dimensional doubly stochastic Poisson process with intensity process $\left\{\Lambda_{t}\right\}$ and that mean $E\left(\Lambda_{t}\right)$ and covariance matrix $K_{\Lambda}(t, u)$ are known. Let $\Lambda_{t}^{l}$ be a linear estimate of $\Lambda_{t}$ in terms of output data $\left\{\boldsymbol{N}_{t}\right\}$ given by

$$
\Lambda_{t}^{l}=\boldsymbol{a}_{t}+\int_{t 0}^{t} H(t, u) d \boldsymbol{N}_{u},
$$

where $\boldsymbol{a}_{t}$ is $n$-dimensional vector and $H(t, u)$ is $n \times n$ impulse response matrix. Then, following assertion holds by [3].

ASSERTION 3 (estimation of $\Lambda_{t}$.) Let the states equation

$$
d \boldsymbol{x}_{t}=A_{t} \boldsymbol{x}_{t} d t+B_{t} d \boldsymbol{\beta}_{t}, \quad \boldsymbol{x}_{t_{0}}=\boldsymbol{x}_{0},
$$

where $A_{t}$ is known $m \times m$ matrix, $B_{t}$ is known $m \times r$ matrix, $\left\{\boldsymbol{\beta}_{t}\right\}$ is $r$-dimensional Brownian motion process and $\boldsymbol{x}_{0}$ is random variable with zero-mean and known covariance matrix $\Sigma_{0}$, satisfy

$$
\mathrm{E}\left(\boldsymbol{x}_{t}\right)=\mathbf{0}, \quad \text { for } t \geqq t_{0}
$$

and

$$
K_{A}(t, u)=C_{t} \mathrm{E}\left(\boldsymbol{x}_{t} \boldsymbol{x}_{u}^{\prime}\right) C_{u}^{\prime}, \quad \text { for } t, u \geqq t_{0},
$$

where $C_{t}$ is known $n \times m$ matrix, that is, $\Lambda_{t}=\mathrm{E}\left(\Lambda_{t}\right)+C_{t} \boldsymbol{x}_{t}$. Denote the estimate $\Lambda_{t}^{l}$ that minimizes error covariance matrix $\mathrm{E}\left[\left(\Lambda_{t}-\Lambda_{t}^{l}\right)\left(\Lambda_{t}-\Lambda_{t}^{l}\right)^{\prime}\right]$ by $\Lambda_{t}^{*}$. Then, the estimate $\Lambda_{t}^{*}$ is given by following equations:

$$
\begin{aligned}
d \boldsymbol{x}_{t}^{*} & =A_{t} \boldsymbol{x}_{t}^{*} d t+\Sigma_{t} C_{t}^{\prime} \operatorname{diag}\left(\mathrm{E}\left(\lambda_{t}^{(i)}\right)\right)^{-1}\left[d \boldsymbol{N}_{t}-\Lambda_{t}^{*} d t\right], \quad \boldsymbol{x}_{t_{0}}^{*}=\mathbf{0}, \\
d \Sigma_{t} & =\left(A_{t} \Sigma_{t}+\Sigma_{t} A_{t}^{\prime}+B_{t} B_{t}^{\prime}-\Sigma_{t} C_{t}^{\prime} \operatorname{diag}\left(\mathrm{E}\left(\lambda_{t}^{(i)}\right)\right)^{-1} C_{t} \Sigma_{t}\right) d t, \quad \Sigma_{t_{0}}=\Sigma_{0}, \\
\Lambda_{t}^{*} & =\mathrm{E}\left(\Lambda_{t}\right)+C_{t} \boldsymbol{x}_{t}^{*} .
\end{aligned}
$$

Furthermore, error covariance matrix of $\Lambda_{t}^{*}$ is given by

$$
\mathrm{E}\left[\left(\Lambda_{t}-\Lambda_{t}^{*}\right)\left(\Lambda_{t}-\Lambda_{t}^{*}\right)^{\prime}\right]=C_{t} \Sigma_{t} C_{t}^{\prime} .
$$

The assertion is shown in [3] as follows.

The estimate. $\Lambda_{t}^{l}$ in the form of (3.16) that minimizes error covariance matrix $\mathrm{E}\left[\left(\Lambda_{t}-\Lambda_{t}^{l}\right)\left(\Lambda_{t}-\Lambda_{t}^{l}\right)^{\prime}\right]$ is given by

$$
\Lambda_{t}^{*}=\mathrm{E}\left(\Lambda_{t}\right)+\int_{t 0}^{t} H_{0}(t, u)\left[d \boldsymbol{N}_{u}-\mathrm{E}\left(\Lambda_{u}\right) d u\right],
$$

where the optimum impulse response matrix $H_{0}(t, u)$ satisfies

$$
H_{0}(t, u) \operatorname{diag}\left(\mathrm{E}\left(\lambda_{u}^{(i)}\right)\right)+\int_{t_{0}}^{t} H_{0}(t, \sigma) K_{\Lambda}(\sigma, u) d \sigma=K_{\Lambda}(t, u), \text { for } t_{0} \leqq u<t .
$$

Seeing (3.19) and (3.20), two intensity processes lead to the same linear intensity estimator if their means and covariance matrices are identical. So we can give outside stochastic process of $A_{t}$ by $\left\{\boldsymbol{x}_{t}\right\}$ that satisfies states equation (3.17). Now, we give an observed process of the states by 


$$
\boldsymbol{r}_{t}=\Lambda_{t}+\operatorname{diag}\left(\mathrm{E}\left(\lambda_{t}^{(i)}\right)\right)^{1 / 2} \boldsymbol{w}_{t},
$$

where $\boldsymbol{w}_{t}$ is $n$-dimensional normal white noise and independent of $\Lambda_{t}$ such that $E\left(\boldsymbol{w}_{t}\right)=\mathbf{0}$ and $E\left(\boldsymbol{w}_{t} \boldsymbol{w}_{-}\right)=\boldsymbol{I}_{n} \delta(t-\tau)$. Then, it is shown that mean and covariance matrix of $\boldsymbol{r}_{t} d t$ and those of $d \boldsymbol{N}_{t}$ are identical by the same way as Section 3.1. Therefore, we can use observed value $d \boldsymbol{N}_{t}$ as $\boldsymbol{r}_{t} d t$. Thus, applying Kalman filtering theory to (3.17) and (3.21), we have equations (3.18).

\section{Analyses of physiological data}

Applying the method stated in the preceeding sections, we try to analyze physiological impulse data [5] which were obtained from the monkey in sexual behavior at Oomura Laboratory of Physiology, Kyushu University, in January 1981. See Fig. 1.

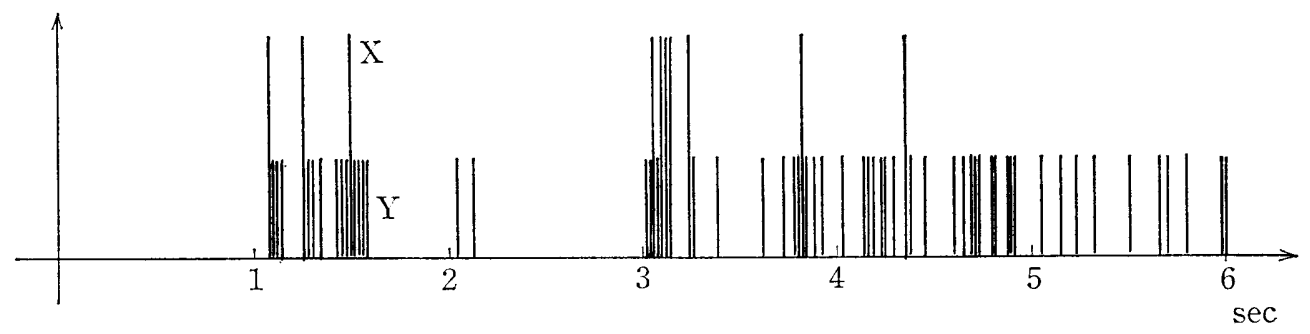

Fig. 1. Impulse sequence in physiological experiment. See [5].

As shown in Fig. 1, two impulse sequences, large and small ones, called $X$ and $Y$ respectively, were simultaneously recorded from a neuron in preoptic area by a microelectrode during 5 minutes. In the figure, impulse sequence for first six seconds is shown. The large impulses were action potentials of the neuron inserted by the electrode and the small impulses corresponded to action potentials of neighboring neuron. We expected that there were some synaptic connections between the neighboring two neurons. However it was difficult to display any connection organically through broken neurons.

Then, following two connections were assumed between the neighboring two neurons firing large impulse sequence $X$ and small impulse sequence $Y$, respectively.

(1) $Y$ is input point process $\left\{M_{t}\right\}$ and $X$ is output point process $\left\{N_{t}\right\}$.

(2) $X$ is input point process $\left\{M_{t}\right\}$ and $Y$ is output point process $\left\{N_{t}\right\}$.

In each case, estimation of parameters in states equation is performed and then using the estimated parameters and output data $\left\{N_{t}\right\}$, we obtain estimates $\left\{\pi_{t}^{*}\right\}$.

Lastly, regarding each $X$ and $Y$ as $\left\{N_{t}\right\}$ with uniformly distributed independent process $\left\{\lambda_{t}\right\}$, we obtain estimates $\left\{\lambda_{t}^{*}\right\}$ in terms of $\left\{N_{t}\right\}$. Then $\left\{\lambda_{t}^{*}\right\}$ are compared with impulse frequency (impulse numbers per second) of $\left\{N_{t}\right\}$.

4.1. The case with $X=\left\{N_{t}\right\}$ and $Y=\left\{M_{t}\right\}$.

Assertion 2 is applied for estimation of parameters. Equations (3.10) and (3.11) are reduced to

$$
\begin{aligned}
d \pi_{t} & =a_{t} \pi_{t} d t+b_{t} d t+u_{t} d t, \\
\tilde{\pi}_{t} & =\pi_{t}+u_{t} .
\end{aligned}
$$


Variances of $u_{t}$ and $w_{t}$ are selected as

$$
V_{u}(t)=1, \quad V_{w}(t)=\frac{1}{4} .
$$

Observed value $\tilde{\pi}_{t}$ is considered as impulse frequency of $Y$.

Then, we obtained estimates $a_{t}^{*}$ and $b_{t}^{*}$ giving perturbation to error covariance matrix $\Sigma_{t}$ by resetting their diagonal elements at 1.0 and non-diagonal elements at 0.0 every 10 seconds (100 steps). Estimates $a_{t}^{*}$ and $b_{t}^{*}$ are shown in Table 1.

Table 1. Estimates $a_{t}^{*}$ and $b_{t}^{*}$ of parameters $a_{t}$ and $b_{t}$ in terms of $Y$.

\begin{tabular}{c|rrrrrrrrrr}
\hline$t(\mathrm{sec})$ & \multicolumn{1}{c}{30} & \multicolumn{1}{c}{60} & \multicolumn{1}{c}{90} & 120 & 150 & 180 & 210 & 240 & 270 & \multicolumn{1}{c}{300} \\
\hline$a_{t}^{*}$ & -0.295 & -0.845 & -0.117 & -0.761 & -0.762 & -0.461 & -0.875 & -0.723 & -0.292 & -0.192 \\
$b_{t}^{*}$ & 1.497 & 1.935 & 2.542 & 2.923 & 2.911 & 3.140 & 2.974 & 3.039 & 2.856 & 2.793 \\
\hline
\end{tabular}

Next, fixing the estimates $a_{t}^{*}$ and $b_{t}^{*}$, we perform estimation of $\pi_{t}$ in terms of $X$ by Assertion 1 in Section 3.1. Equations (3.1) and (3.2) are given by
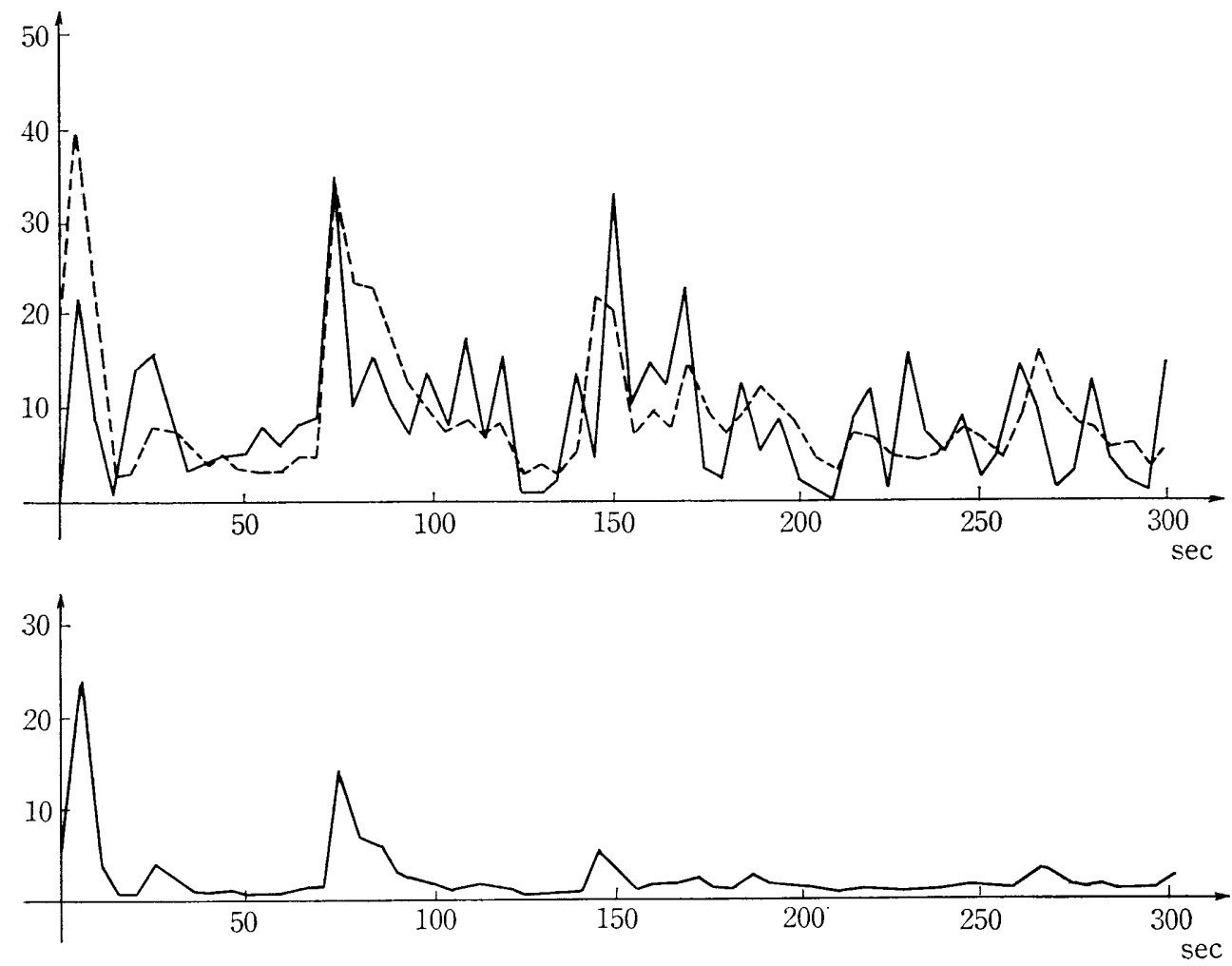

Fig. 2. Estimate $\pi_{t}^{*}$ compared with impulse frequency of $Y$.

Upper: solid line shows impulse frequency of $Y$ and broken line shows estimate $\pi_{t}^{*}$. Lower: mean-square error $\Sigma_{t}$ of $\pi_{t}^{*}$. 


$$
\begin{aligned}
d \pi_{t} & =a_{t}^{*} \pi_{t} d t+b_{t}^{*} d t+u_{t} d t, \\
\lambda_{t} & =0.2 \pi_{t} .
\end{aligned}
$$

As the observation of $\lambda_{t}$, we use impulse frequency of $X$. Estimate $\pi_{t}^{*}$ is calculated and the graph is shown in Fig. 2.

4.2. The case with $X=\left\{M_{t}\right\}$ and $Y=\left\{N_{t}\right\}$.

In this case, numerical calculation is carried out by the same way as Section 4.1, except the exchange, $X=\left\{M_{t}\right\}, Y=\left\{N_{t}\right\}$, and $\lambda_{t}=5.0 \pi_{t}$. Estimates $a_{t}^{*}$ and $b_{t}^{*}$ are shown in Table 2. Estimate $\pi_{t}^{*}$ is shown in Fig. 3.

Table 2. Estimates $a_{t}^{*}$ and $b_{t}^{*}$ of parameters $a_{t}$ and $b_{t}$ in terms of $X$.

\begin{tabular}{c|rrrrrrrrrr}
\hline$t(\mathrm{sec})$ & \multicolumn{1}{|c}{30} & 60 & 90 & 120 & 150 & 180 & 210 & 240 & 270 & 300 \\
\hline$a_{t}^{*}$ & -0.521 & -0.637 & -0.467 & -0.682 & -0.540 & -0.389 & -0.462 & -0.597 & -0.643 & -0.609 \\
$b_{t}^{*}$ & 0.532 & 0.633 & 0.610 & 0.684 & 0.753 & 0.838 & 0.806 & 0.824 & 0.577 & 0.353 \\
\hline
\end{tabular}
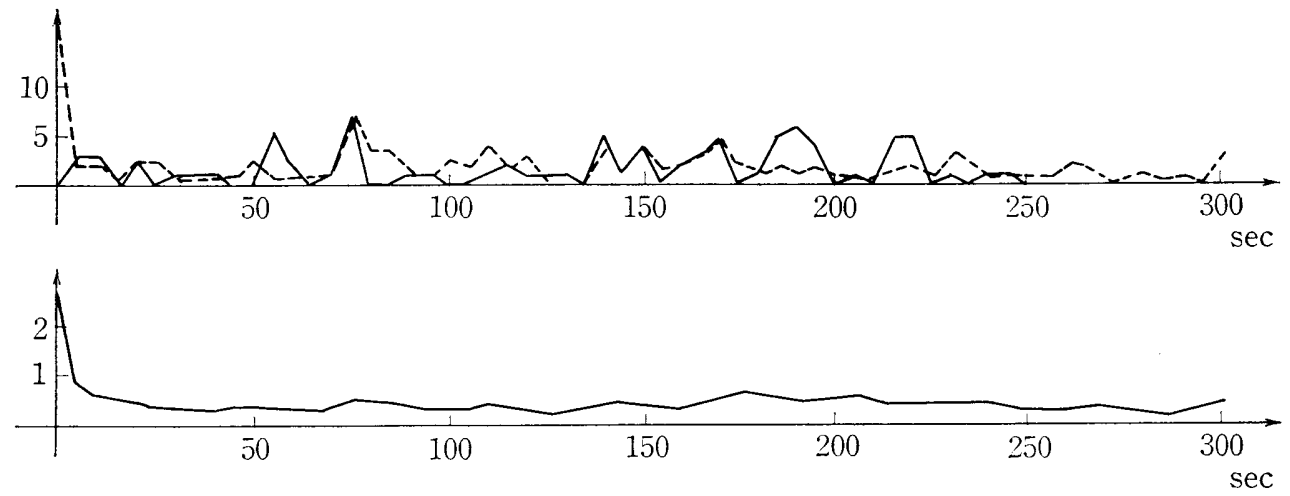

Fig. 3. Estimate $\pi_{t}^{*}$ compared with impulse frequency of $X$.

Upper: solid line shows impulse frequency of $X$ and broken line shows estimate $\pi_{t}^{*}$. Lower: mean-square error $\Sigma_{t}$ of $\pi_{t}^{*}$.

As compared Fig. 3 with Fig. 2, a question arise: which is better model 4.1 or 4.2 to explain the physiological data in Fig. 1? To see some significant differences between two models, we need more experimental facts and analytical study. But, since intensity processes $\left\{\pi_{t}\right\}$ satisfying the stochastic differential equation (3.1) are Markov processes, models of nervous systems given in 4.1 and 4.2 may be called Marcov nervous systems.

\subsection{Estimation of $\lambda_{t}$ in terms of $\left\{N_{t}\right\}$.}

We apply Assertion 3 in Section 3.3 for each $X$ and $Y$ which is considered as $\left\{N_{t}\right\}$.

In this case, Kalman filterting theory is applied only for a method of estimating $\lambda_{t}$ in terms of $\left\{N_{t}\right\}$ without assuming any structure of nervous system. Then, stochastic process $\left\{\lambda_{t}\right\}$ is considered as independent stochastic process. This section was selected as a simple control model for the modeling of nervous systems in Section 4.1 and 4.2.

At first, in the case of $X=\left\{N_{t}\right\}, \lambda_{t}$ is given as follows. 


$$
\lambda_{t}=0.9 \alpha_{t},
$$

where $\left\{\alpha_{t}\right\}$ is independent stochastic process and uniformly distributed in $[0,8]$. Then, we have

$$
\mathrm{E}\left(\lambda_{t}\right)=3.6
$$

and

$$
K_{\lambda}(t, u)= \begin{cases}4.32, & t=u \\ 0, & t \neq u .\end{cases}
$$

Coefficients $a_{t}$ and $b_{t}$ in applied equation (3.17) and $c_{t}$ are calculated as

$$
a_{t}=2.0, \quad b_{t}=1.0, \quad c_{t}=\frac{7.2}{\sqrt{ } 3} .
$$

Then estimate $\lambda_{t}^{*}$ is obtained and shown in Fig. 4.
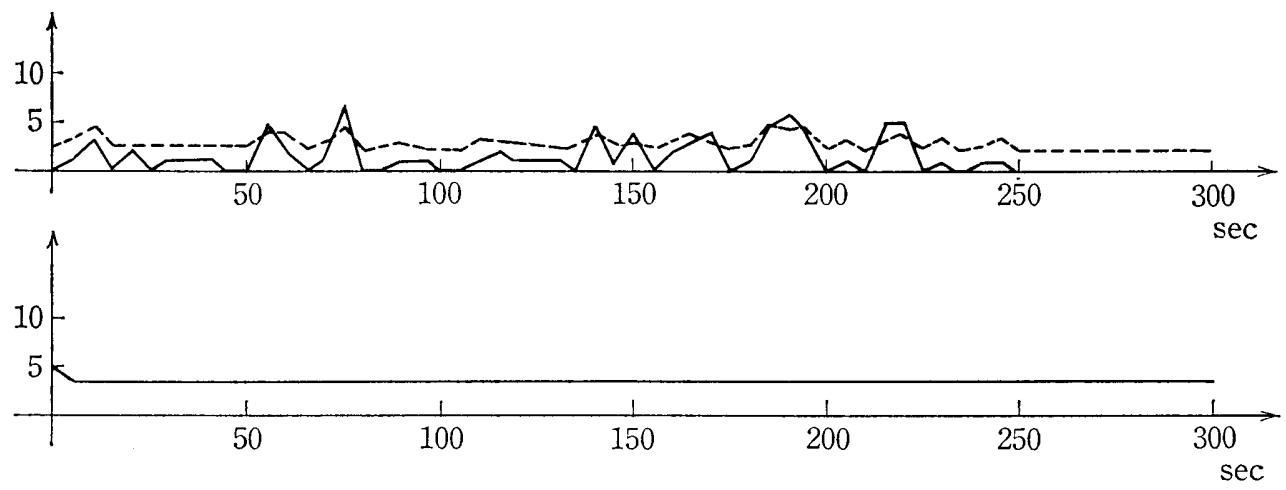

Fig. 4. Estimate $\lambda_{t}^{*}$ obtained in terms of $X$ and compared with impulse frequency of $X$. Upper: solid line shows impulse frequency of $X$ and broken line shows estimate $\lambda_{t}^{*}$. Lower: mean-square error $\Sigma_{t}$ of $\lambda_{t}^{*}$.

In the case of $Y=\left\{N_{t}\right\}, \lambda_{t}^{*}$ is obtained similarly except that

$$
\begin{aligned}
\lambda_{t} & =5.0 \alpha_{t}, \\
\mathrm{E}\left(\lambda_{t}\right) & =20 \\
K_{\lambda}(t, u) & = \begin{cases}\frac{400}{3}, & t=u \\
0, & t \neq u,\end{cases}
\end{aligned}
$$

and coefficients

$$
a_{t}=2.0, \quad b_{t}=1.0, \quad c_{t}=\frac{40}{\sqrt{3}} .
$$

The result is shown in Fig. 5.

Comparing Fig. 4 and Fig. 5 with Fig. 3 and Fig. 2, respectively, the models in 

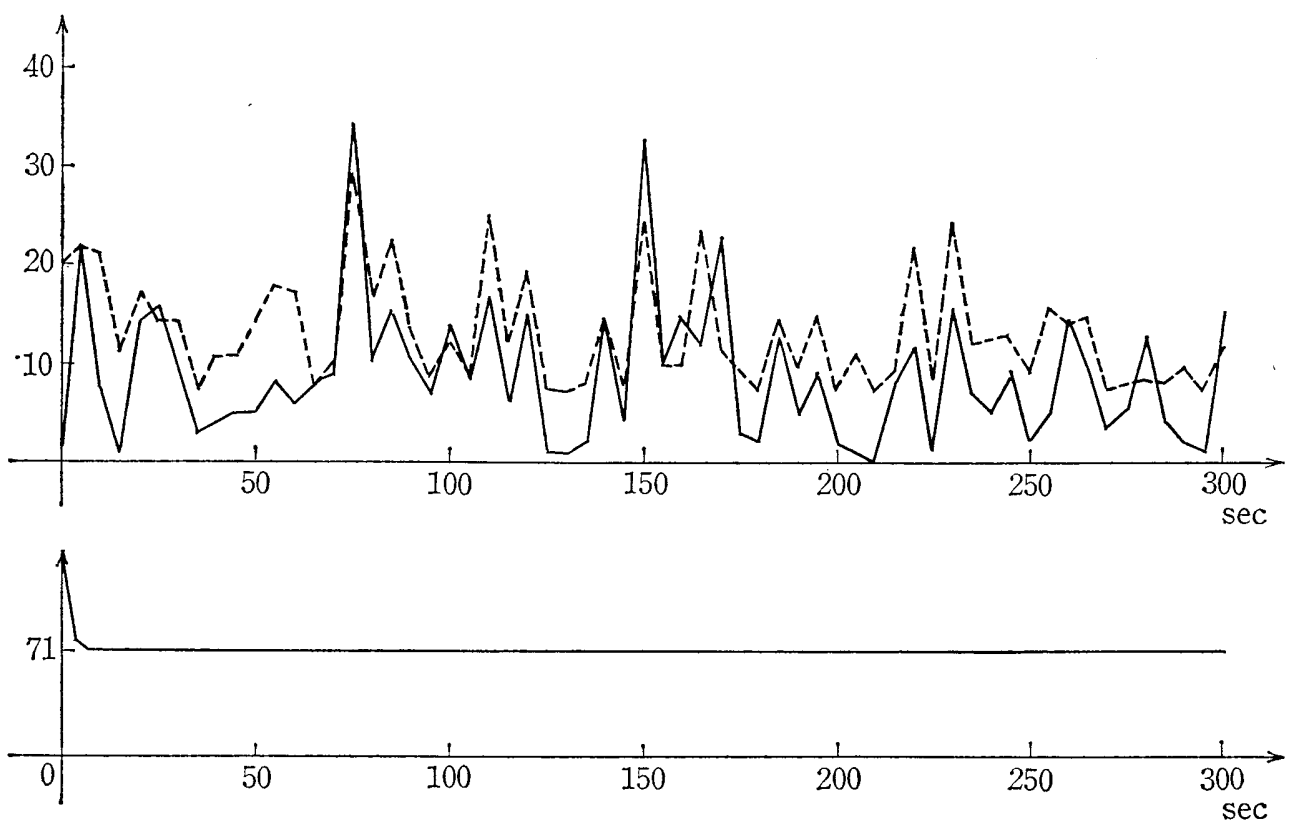

Fig. 5. Estimate $i_{t}^{*}$ obtained in terms of $Y$ and compared with impulse frequency of $Y$. Upper: solid line shows impulse frequency of $Y$ and broken line shows estimate $\lambda_{i}^{*}$. Lower: mean-square error $\Sigma_{t}$ of $\lambda_{t}^{*}$.

4.1 and 4.2 are better than the models in 4.3 to describe the physiological data in Fig. 1 , as we expected.

\section{Results and Problems}

In this paper, we gave some methods of mathematical modeling and analyses of nervous systems by following two lines.

(1) Neuron impulse sequences are represented by doubly stochastic Poisson processes.

(2) The structure of nervous system is described by stochastic differential equations in which random variables are intensity processes.

Then, experimental data recorded from a neuron in the nervous system, preoptic area of monkey, were analyzed by given methods. The system equations were selected to obtain minimum error variance. Good fitting of the estimates to data showed effective modeling. However, when we try to form a model accounting some functions of a nervous system, following questions arise.

(1) Why are a great number of neurons, of $10^{3}$ or $10^{4}$, needed for an area which controls single function of a brain? How are these neurons mutually related?

(2) How are many control areas in brain mutually related and integrated to a function?

Basic principle of the two interrelations may be identical each other, that is, neural network within an area may operate similarly to that between many areas. Methods of multi-dimensional doubly stochastic Poisson processes and of simultaneous stochastic 
differential equations, both stated in this paper, may be effective for each study of the two interrelations.

There are some problems in the study.

(1) More than three impulse sequences which are simultaneously recorded in physiological experiments are needed.

(2) A method in which some basic networks between two or three neurons are connected for increasing the functions of their networks, must be attacked.

(3) Modeling of nervous systems is to be studied in connection with changes of neuron impulse patterns caused by animal behavior, that is, taking food, learning and so on.

Experiments designed on the solutions of these problems may give us interesting data forming multi-dimenisonal neuron impulse sequence. We believe that the method given in this paper becomes powerful tool to analyze these data and to explain advanced functions of nervous systems.

\section{Acknowledgement}

The authors wish to express their gratitude to Professor Y. Oomura for the use of physiological data obtained in his laboratory.

\section{References}

[1] Wiener, N., Nonlinear Problems in Random Theory, M.I.T. Press, Cambridge, 1958.

[2] Marmarelis, P.Z. and NaKa, Ken-ichi, White-noise analysis of neuron chain: An application of the Wiener theory, Science, 175, (Mar. 1972), 1276-1278.

[3] Snyder, D. L., Random Point Processes, Wiely, New York, 1975.

[4] Arimoto, S., Kalman Filter, Sangyō Tosho, 1977.

[5] Oomlra, Y., Yoshimatsu, H. and Ao, S., Preoptic area: Does the neural activity reflect motivation for sexual behavior in the monkey?, Science, submitted. 